\title{
AVALIAÇÃO E CONTROLE DE INSTRUMENTAIS UTILIZADOS EM SALA OPERATÓRIA DURANTE CIRURGIAS TORÁCICAS
}

Assessment and control of instruments utilized in operating room during thoracic surgeries Evaluación y control de instrumentales utilizados en quirófano durante cirugías torácicas

Vítor Marraschi ${ }^{1}$, Amanda Cristina Cocco ${ }^{1}$, Adrielly Raymundo Gaspar', Cleuza Aparecida Vedovato ${ }^{2}$, Ana Paula Boaventura ${ }^{3}$

RESUMO: Objetivo: Avaliar o número de instrumentais cirúrgicos não utilizados durante as cirurgias torácicas realizadas em um hospital universitário. Métodos: Trata-se de um estudo exploratório, descritivo, transversal com abordagem quantitativa, realizado a partir do levantamento de dados sobre a utilização ou não de instrumentais cirúrgicos presentes nas caixas cirúrgicas. Resultados: Foram observadas 30 cirurgias torácicas, sendo a média de instrumentais utilizados por cirurgia de $84,53 \%$ e a média de instrumentais não utilizados de 15,48\%. Conclusão: São necessárias reformulações na composição das caixas cirúrgicas dessa especialidade a fim de otimizar a utilização e o processamento dos instrumentais.

Palavras-chave: Instrumentos cirúrgicos. Controle de custos. Procedimentos cirúrgicos operatórios. Cirurgia torácica. Enfermagem perioperatória.

ABSTRACT: Objective: To assess the number of unused surgical instruments during thoracic surgeries performed at a university hospital. Methods: An exploratory, descriptive, cross-sectional study with a quantitative approach, conducted by gathering data on the use or lack of use of surgical instruments present in the surgical box. Results: A total of thirty thoracic surgeries were observed, with a mean of $84.53 \%$ of instruments utilized for surgery and a mean of $15.48 \%$ of instruments left unused. Conclusion: A reconfiguration of the surgical boxes for this specialty is needed in order to optimize the utilization and the process of these instruments.

Keywords: Surgical instruments. Cost control. Surgical procedures. Operative procedures. Thoracic surgery. Perioperative nursing.

RESUMEN: Objetivo: Evaluar el número de instrumentales quirúrgicos no utilizados durante las cirugías torácicas realizadas en un hospital universitario. Métodos: Se trata de un estudio exploratorio, descriptivo, transversal con abordaje cuantitativo, realizado a partir del levantamiento de datos sobre a utilización o no de instrumentales quirúrgicos presentes en las cajas quirúrgicas. Resultados: Fueron observadas 30 cirugías torácicas, siendo el promedio de instrumentales utilizados por cirugía del $84,53 \%$ y el promedio de instrumentales no utilizados del 15,48\%. Conclusión: Son necesarias reformulaciones en la composición de las cajas quirúrgicas de esa especialidad a fin de optimizar la utilización y el procesamiento de los instrumentales. Palabras clave: Instrumentos quirúrgicos. Control de costos. Procedimientos quirúrgicos operativos. Cirugía torácica. Enfermería perioperatoria.

\footnotetext{
Alunos do curso de graduação em Enfermagem da Faculdade de Enfermagem da Universidade Estadual de Campinas (UNICAMP) - Campinas (SP), Brasil. Enfermeira mestre da Faculdade de Enfermagem da UNICAMP - Campinas (SP), Brasil.

${ }^{3}$ Professora doutora da Faculdade de Enfermagem da UNICAMP - Campinas (SP), Brasil. E-mail: apboa@unicamp.br

Avenida José Puccinelli, 10 (Rua 6, casa 92) - Cascata - CEP: 13146-000 - Paulínia (SP), Brasil.

Recebido: 28 nov. 2016 - Aprovado: 22 mar. 2017

DOI: $10.5327 / 21414-4425201700030002$
} 


\section{INTRODUÇÃO}

O Centro Cirúrgico (CC) é uma unidade hospitalar na qual são realizadas cirurgias de alta, média e baixa complexidade e, portanto, requer equipe bem treinada e qualificada. Recomenda-se que o CC esteja interligado com a Unidade de Terapia Intensiva, a Sala de Recuperação Pós-anestésica e o Pronto Socorro para facilitar o atendimento de emergência, também devendo estar próximo ao Centro de Material e Esterilização (CME), a fim de facilitar o fluxo de materiais esterilizados ${ }^{1}$.

A CME é definida como uma área para recepção, expurgo, preparo, esterilização, guarda e distribuição de materiais esterilizados para as unidades do hospital. Deve ter acesso físico restrito ao pessoal lotado no serviço e possuir controle de temperatura e umidade para manter a esterilidade dos materiais processados, evitando o crescimento bacteriano e a deterioração do material estéril ${ }^{1,2}$.

A partir do momento em que é utilizado nas cirurgias, o material é considerado contaminado e encaminhado para a CME, onde será submetido ao processamento que o tornará estéril novamente. Vale lembrar que, conforme a Resolução da Diretoria Colegiada (RDC) $\mathrm{n}^{\circ}$ 30, de 15 de fevereiro de 2006, da Agência Nacional de Vigilância Sanitária (ANVISA), é proibido reesterilizar os materiais, devendo, mesmo aqueles que foram apenas abertos e não utilizados, ser reprocessados, ou seja, passar novamente por todo o processo, desde a limpeza até o armazenamento ${ }^{3,4}$.

Existe um vasto acervo de instrumentais cirúrgicos desenvolvidos para atender às novas técnicas cirúrgicas, com as funções de auxiliar, facilitar e promover precisão aos cirurgiões. Esses instrumentais são distribuídos em grupos conforme seu uso e suas funções durante cada tempo do ato cirúrgico, sendo eles divididos em especiais e básicos ou comuns. Os especiais são utilizados somente em alguns tempos de determinadas cirurgias, ou seja, são instrumentais específicos. Os comuns são os instrumentais básicos que compõem todas as caixas cirúrgicas e podem ser usados em qualquer tipo de intervenção, tendo as seguintes funções: diérese, como a lâmina de bisturi e as tesouras; hemostasia, como a pinça Kelly; preensão, como a pinça Allis e as pinças de campo ou Backhaus; separação, como os afastadores; e síntese, como os porta-agulhas ${ }^{3,5-7}$.

Por fim, existem alguns exemplos de instrumentos utilizados especificamente para algumas especialidades cirúrgicas, como a pinça Abadie, empregada em cirurgias do trato digestório, ou o amigdalótomo de Sluder-Ballenger, usado em cirurgias de amígdalas ${ }^{7}$.
Os instrumentais cirúrgicos utilizados no ambiente hospitalar são tidos como recursos materiais e têm extrema importância dentro de uma instituição, com fins lucrativos ou não, pois representam $75 \%$ do capital dos estabelecimentos de assistência à saúde; portanto, a forma de administrá-los reflete diretamente nos custos hospitalares. Logo, o excesso de instrumentais processados e não utilizados pode resultar no aumento de custos, além da depreciação, deterioração e desperdício dos mesmos. Para que isso não ocorra, os instrumentais não devem estar em excesso nas caixas e mesas cirúrgicas, e sim estar presentes apenas os indispensáveis ou comprovadamente úteis para a intervenção cirúrgica proposta ${ }^{6,8}$.

Na avaliação de custos, é de extrema importância destacar que o produto final, no caso, o material hospitalar esterilizado, refere-se à ação de três fatores inter-relacionados: materiais utilizados, mão de obra e tecnologia empregada para o processamento. Esses fatores, se bem administrados, não produzem prejuízo em si, e norteiam expectativas para a redução dos custos, mantendo a qualidade da assistência. Para tanto, deve-se ter uma administração de qualidade e atenta a esses fatores ${ }^{9}$.

Para chegar a esse valor dos custos com esterilização, realiza-se a contabilização de gastos com insumos, tempo e mão de obra utilizados no processo, desde a lavagem de cada instrumento até a estocagem do material na CME, avaliando-se ainda a tecnologia empregada para a realização do mesmo incluindo nesse último item a manutenção da esterilizadora e a energia elétrica gasta no processo ${ }^{9,10}$.

Em estudo realizado em 2015, observou-se o valor de $\mathrm{R} \$ 0,29$ por instrumental processado, identificando-se em apenas 17 cirurgias de médio porte o custo de $\mathrm{R} \$ 1.584,17 \mathrm{em}$ um mês com a esterilização de instrumentais que não foram utilizados nas cirurgias, mas que compõem a caixa cirúrgica ${ }^{3}$.

As cirurgias torácicas constituem importante especialidade, uma vez que oferecem intervenções nos pulmões, pleuras, mediastino e parede torácica integrada a uma equipe médica especializada de infectologistas, oncologistas e pneumologistas; nesse contexto o enfermeiro atua no processo de enfermagem perioperatório complexo dessa especialidade em cirurgias como toracotomia, broncoscopia e lobectomia, entre outras 5 .

Um procedimento torácico inclui a combinação de instrumentais delicados e pesados utilizados em incisões, dissecações e retrações, bem como para cortar e fixar tecidos e vasos no tórax, além de facilitarem a inspeção e a intervenção nas estruturas torácicas. Para isso, o enfermeiro prevê e provê esses instrumentais antes da cirurgia, evitando desperdícios; garante sua utilização adequada e precisa durante todo o procedimento, que geralmente envolve incisões de longa duração; 
e, por fim, antes do fechamento, garante que todos os instrumentais sejam minuciosamente conferidos e contados ${ }^{5}$.

Logo, o gerenciamento dos materiais em uma sala operatória $(\mathrm{SO})$ é tarefa do enfermeiro que atua no CC, e é parte fundamental da assistência de enfermagem perioperatória, que envolve os cuidados e a segurança do paciente nos períodos pré, trans e pós-operatórios ${ }^{1-3}$.

Para uma cirurgia bem elaborada e com êxito no seu desfecho, não deve haver instrumentais em excesso nas caixas e mesas cirúrgicas. Devem estar presentes apenas os instrumentos indispensáveis ou comprovadamente úteis para a realização do procedimento. Assim, a pergunta principal deste estudo é: "Todos os instrumentais que compõem as caixas cirúrgicas da especialidade "cirurgia do tórax" são utilizados em SO?".

Os resultados deste trabalho contribuirão para o aperfeiçoamento das práticas de enfermagem perioperatória no que se refere à avaliação e ao controle de instrumentais em $\mathrm{SO}$, além de fornecerem subsídios para o controle de custos com o processamento de instrumentais no CME.

\section{OBJETIVO}

Avaliar o número de instrumentais cirúrgicos utilizados e não utilizados que compõem as caixas cirúrgicas das cirurgias torácicas.

\section{MÉTODOS}

Trata-se de um estudo descritivo, observacional, transversal, com abordagem quantitativa, realizado em um hospital universitário da cidade de Campinas (SP). O hospital, de nível terciário e quaternário, é integralmente financiado pelo Sistema Único de Saúde (SUS) e possui 403 leitos, onde todos os atendimentos são realizados e pagos integralmente pelo SUS. O CC realiza em média 200 cirurgias por mês, distribuídas em 12 SOs destinadas às cirurgias eletivas e $4 \mathrm{SOs}$ destinadas às cirurgias de emergência. A pesquisa foi aprovada pelo Comitê de Ética em Pesquisa da Universidade Estadual de Campinas, sob o parecer consubstanciado de número 1.384.178, de 6 de janeiro de 2016 .

A coleta dos dados foi realizada de fevereiro a abril de 2015, autorizada pela diretoria de enfermagem do CC e pelos enfermeiros responsáveis pelo local após a explicação dos objetivos da pesquisa, com leitura e assinatura do Termo de Consentimento Livre e Esclarecido. Dessa forma, foram garantidos os preceitos éticos e legais envolvidos em pesquisa com seres humanos contidos na Resolução 466/2012 do Conselho Nacional de Saúde ${ }^{11}$.

O cálculo amostral foi realizado considerando-se o objetivo de estimar a proporção de instrumentais cirúrgicos não utilizados nas cirurgias torácicas de fevereiro a abril de 2015. No cálculo amostral, foi considerada uma proporção $p$ igual a 0,50, cujo valor representa a variabilidade máxima da distribuição binomial, gerando assim uma estimativa com o maior tamanho amostral possível.

A população $(\mathrm{N})$ considerada para o cálculo do tamanho amostral foi composta de 3.195 cirurgias de todas as especialidades, realizadas de fevereiro a abril de 2015 , das quais 336 eram cirurgias torácicas. Além disso, foi assumido erro amostral de $5 \%$ e nível de significância de $5 \%$. Com isso, o tamanho amostral total calculado foi de 343 cirurgias. Essa amostra foi dividida proporcionalmente de acordo com o número e as especialidades das cirurgias realizadas. Para as cirurgias torácicas, a amostra calculada foi de 30 cirurgias.

Foram especificados no instrumento de coleta: o nome da cirurgia, as caixas cirúrgicas envolvidas, a data e o número da cirurgia, a identificação nominal dos instrumentais mais comuns utilizados em cirurgias - divididos em diérese, hemostasia, síntese, outras —; duas colunas indicando a quantidade de cada peça na caixa e o número de peças que ficaram fora da mesa ao final da cirurgia; e, por fim, a soma dos valores das duas colunas e um espaço para observações cirúrgicas.

A maior parte das cirurgias necessitava de mais de uma caixa para sua realização, de forma que cada caixa vinda da CME era acompanhada de uma listagem com os instrumentais nela presentes. Para a coleta de dados, o pesquisador entrava na SO durante a cirurgia e, com o auxílio dessas listagens, preenchia a primeira parte do instrumento de coleta de dados, informando quais instrumentos estavam presentes, bem como sua quantidade. Ao final da cirurgia, o pesquisador entrava novamente na $\mathrm{SO}$ e preenchia a segunda parte do instrumento, registrando o número de instrumentos cirúrgicos que permaneciam intactos nas caixas vindas do CME, ou seja, aqueles que sequer chegaram à mesa cirúrgica.

No instrumento de coleta de dados de cada cirurgia, foi realizada a divisão dos instrumentais cirúrgicos conforme os tempos cirúrgicos: diérese, hemostasia, auxiliares, síntese e outros (enquadrando principalmente os instrumentos envolvidos na exérese). Esse processo foi implementado em 30 cirurgias torácicas. Consideraram-se para este trabalho apenas as cirurgias realizadas pela especialidade de "cirurgia do tórax", que, nessa instituição, realiza as traqueostomias. 


\section{RESULTADOS}

Do total de 30 cirurgias torácicas analisadas, foram observados 3.333 instrumentais, dos quais $516(15,48 \%)$ não foram utilizados. A média de instrumentais utilizados por cirurgia foi de 111,1, e a média de instrumentais não utilizados foi de 17,87 (Tabela 1).

O percentual de instrumentais não utilizados nas cirurgias torácicas corresponde a 15,48\% (17,87), em 94 caixas observadas nesses 30 procedimentos.

Foram realizadas cirurgias de pequeno, médio e grande porte, sendo: 8 traqueostomias $(26,6 \%), 4$ traqueoplastias (13,33\%), 6 pleuroscopias (20\%), 4 mediastinoscopias (13,3\%), 3 lobectomias (10,0\%) e 5 costectomias (16,66\%), que utilizaram de 1 a 12 caixas cirúrgicas. A Tabela 2 apresenta a distribuição da utilização de caixas e instrumentais dessa especialidade por porte cirúrgico e por cirurgias propostas.

Os percentuais de instrumentais utilizados por tempo cirúrgico nessas cirurgias torácicas foram: diérese, 13,17\%; hemostasia, 16,14\%; auxiliares, 13,49\%; síntese, 11,41\%; e outros instrumentais, 19,39\% (Tabela 3).

Destaca-se o maior número médio de instrumentais não utilizados para hemostasia $(33,30)$ e outros $(39,73)$ instrumentais

Tabela 1. Distribuição de instrumentais e caixas cirúrgicas das cirurgias torácicas. Campinas, 2016. $(n=30)$.

\begin{tabular}{|l|c|c|c|c|}
\hline Variável & Média & $\begin{array}{c}\text { Desvio- } \\
\text { padrão }\end{array}$ & Mínimo & Máximo \\
\hline $\begin{array}{l}\text { Instrumentais } \\
\text { utilizados }\end{array}$ & 111,1 & 93,03 & 19,0 & 424,0 \\
\hline $\begin{array}{l}\text { Instrumentais } \\
\text { não utilizados }\end{array}$ & 17,87 & 22,66 & 0,0 & 92,0 \\
\hline $\begin{array}{l}\text { Número } \\
\text { de caixas } \\
\text { utilizadas }\end{array}$ & 3,13 & 2,83 & 1,0 & 12,0 \\
\hline
\end{tabular}

Tabela 2. Distribuição do total de caixas cirúrgicas e instrumentais por cirurgia. Campinas, 2016.

\begin{tabular}{|l|c|c|c|c|}
\hline \multirow{2}{*}{ Cirurgia } & \multicolumn{4}{|c|}{ Instrumentais } \\
\cline { 2 - 5 } & $\begin{array}{c}\text { Total } \\
\text { caixas }\end{array}$ & $\begin{array}{c}\text { Total } \\
\text { início }\end{array}$ & $\begin{array}{c}\text { Não } \\
\text { usados }\end{array}$ & Usados \\
\hline Grande porte & \multicolumn{3}{|c|}{} \\
\hline Costectomia & 7 & 244 & 55 & 189 \\
\hline Costectomia & 6 & 214 & 53 & 161 \\
\hline Costectomia & 2 & 106 & 4 & 102 \\
\hline Costectomia & 7 & 424 & 30 & 394 \\
\hline Costectomia & 4 & 112 & 0 & 112 \\
\hline Lobectomia & 10 & 220 & 48 & 172 \\
\hline Lobectomia & 12 & 246 & 43 & 203 \\
\hline Lobectomia & 6 & 206 & 0 & 206 \\
\hline Médio porte & & & & \\
\hline Traqueoplastia & 2 & 108 & 0 & 108 \\
\hline Traqueoplastia & 4 & 111 & 16 & 95 \\
\hline Traqueoplastia & 4 & 209 & 82 & 127 \\
\hline Traqueoplastia & 2 & 139 & 23 & 116 \\
\hline Pequeno porte & & & & \\
\hline Mediastinoscopia & 4 & 123 & 14 & 109 \\
\hline Mediastinoscopia & 1 & 37 & 17 & 20 \\
\hline Mediastinoscopia & 2 & 65 & 0 & 65 \\
\hline Mediastinoscopia & 2 & 122 & 12 & 110 \\
\hline Pleuroscopia & 1 & 38 & 5 & 33 \\
\hline Pleuroscopia & 2 & 65 & 10 & 55 \\
\hline Pleuroscopia & 1 & 38 & 6 & 32 \\
\hline Pleuroscopia & 2 & 73 & 0 & 73 \\
\hline Pleuroscopia & 1 & 38 & 5 & 33 \\
\hline Pleuroscopia & 1 & 22 & 2 & 20 \\
\hline Traqueostomia & 1 & 38 & 5 & 33 \\
\hline Traqueostomia & 1 & 115 & 25 & 90 \\
\hline Traqueostomia & 2 & 32 & 6 & 26 \\
\hline Traqueostomia & 1 & 29 & 0 & 29 \\
\hline Traqueostomia & 2 & 64 & 42 & 22 \\
\hline Traqueostomia & 1 & 19 & 1 & 18 \\
\hline Traqueostomia & 1 & 28 & 3 & 25 \\
\hline Tralaeostomia & 2 & 48 & 9 & 39 \\
\hline & 94 & 3333 & 516 & 2817 \\
\hline
\end{tabular}

Tabela 3. Distribuição de instrumentais utilizados e não utilizados por tempos cirúrgicos nas cirurgias torácicas. Campinas, 2016 ( $\mathrm{n}=30$ ).

\begin{tabular}{|c|c|c|c|c|c|}
\hline Tempos cirúrgicos & Instrumentais & Média & Desvio-padrão & Mínimo & Máximo \\
\hline \multirow{2}{*}{ Diérese } & Utilizados & 9,37 & 6,63 & 2,00 & 26,00 \\
\hline & Não utilizados & 1,67 & 2,64 & 0,00 & 10,00 \\
\hline \multirow{2}{*}{ Hemostasia } & Utilizados & 33,30 & 21,77 & 7,00 & 80,00 \\
\hline & Não utilizados & 6,03 & 7,63 & 0,00 & 24,00 \\
\hline \multirow{2}{*}{ Auxiliares } & Utilizados & 20,33 & 14,24 & 6,00 & 57,00 \\
\hline & Não utilizados & 3,07 & 4,39 & 0,00 & 17,00 \\
\hline \multirow{2}{*}{ Síntese } & Utilizados & 8,37 & 7,38 & 1,00 & 25,00 \\
\hline & Não utilizados & 1,53 & 3,76 & 0,00 & 20,00 \\
\hline \multirow{2}{*}{ Outros } & Utilizados & 39,73 & 69,07 & 0,00 & 363,00 \\
\hline & Não utilizados & 5,57 & 9,73 & 0,00 & 32,00 \\
\hline
\end{tabular}


específicos da cirurgia propriamente dita, que também são os instrumentais em maior número na composição dessas caixas cirúrgicas, como pode ser observado na Tabela 4, sendo $999(29,97 \%)$ instrumentais de hemostasia e $1.192(35,7 \%)$ outros instrumentais.

Os instrumentais denominados como "outros" foram contabilizados em maior número porque se referem a instrumentais da especialidade "cirurgia do tórax", mas não da cirurgia proposta, como afastadores, grampeadores, tipos específicos de fórceps, porta-esponjas, coletores de materiais citológicos, retratores e aproximadores, instrumentos para secção óssea, entre outros necessários para a realização de cirurgias torácicas nessa instituição.

\section{DISCUSSÃO}

Em estudo análogo - com objetivo e metodologias semelhantes - realizado em um hospital de pequeno porte no interior do estado de São Paulo, encontrou-se um total de $52 \%$ de instrumentais não utilizados e um custo de R\$ 0,29 por instrumental processado no CME,

Tabela 4. Distribuição dos instrumentais utilizados por tempos cirúrgicos em cada cirurgia. Campinas, 2016.

\begin{tabular}{|c|c|c|c|c|c|c|c|c|c|c|}
\hline \multirow[b]{2}{*}{ Cirurgia } & \multicolumn{2}{|c|}{ Diérese } & \multicolumn{2}{|c|}{ Hemostasia } & \multicolumn{2}{|c|}{ Auxiliares } & \multicolumn{2}{|c|}{ Síntese } & \multicolumn{2}{|c|}{ Outros } \\
\hline & $\begin{array}{l}\text { Total } \\
\text { início }\end{array}$ & $\begin{array}{c}\text { Não } \\
\text { usados }\end{array}$ & $\begin{array}{l}\text { Total } \\
\text { início }\end{array}$ & $\begin{array}{c}\text { Não } \\
\text { usados }\end{array}$ & $\begin{array}{l}\text { Total } \\
\text { início }\end{array}$ & $\begin{array}{c}\text { Não } \\
\text { usados }\end{array}$ & $\begin{array}{l}\text { Total } \\
\text { início }\end{array}$ & $\begin{array}{c}\text { Não } \\
\text { usados }\end{array}$ & $\begin{array}{l}\text { Total } \\
\text { início }\end{array}$ & $\begin{array}{c}\text { Não } \\
\text { usados }\end{array}$ \\
\hline \multicolumn{11}{|l|}{ Grande porte } \\
\hline Costectomia & 26 & 10 & 71 & 11 & 57 & 8 & 25 & 2 & 65 & 24 \\
\hline Costectomia & 23 & 4 & 71 & 18 & 55 & 17 & 24 & 4 & 41 & 10 \\
\hline Costectomia & 7 & 0 & 29 & 0 & 16 & 1 & 12 & 0 & 42 & 3 \\
\hline Costectomia & 11 & 0 & 15 & 0 & 26 & 0 & 9 & 0 & 363 & 30 \\
\hline Costectomia & 7 & 0 & 29 & 0 & 17 & 0 & 15 & 0 & 44 & 0 \\
\hline Lobectomia & 22 & 4 & 46 & 16 & 29 & 7 & 17 & 1 & 106 & 20 \\
\hline Lobectomia & 22 & 5 & 53 & 7 & 38 & 5 & 19 & 4 & 114 & 32 \\
\hline Lobectomia & 19 & 0 & 49 & 0 & 31 & 0 & 14 & 0 & 93 & 0 \\
\hline \multicolumn{11}{|l|}{ Médio porte } \\
\hline Traqueoplastia & 8 & 0 & 45 & 0 & 27 & 0 & 8 & 0 & 20 & 0 \\
\hline Traqueoplastia & 8 & 1 & 31 & 8 & 17 & 3 & 11 & 4 & 44 & 0 \\
\hline Traqueoplastia & 14 & 9 & 80 & 24 & 34 & 15 & 23 & 20 & 58 & 24 \\
\hline Traqueoplastia & 10 & 2 & 55 & 10 & 40 & 2 & 11 & 3 & 23 & 6 \\
\hline \multicolumn{11}{|l|}{ Pequeno porte } \\
\hline Mediastinoscopia & 7 & 1 & 37 & 9 & 21 & 4 & 8 & 0 & 50 & 0 \\
\hline Mediastinoscopia & 5 & 3 & 20 & 11 & 10 & 3 & 2 & 0 & 0 & 0 \\
\hline Mediastinoscopia & 4 & 0 & 20 & 0 & 10 & 0 & 3 & 0 & 28 & 0 \\
\hline Mediastinoscopia & 8 & 0 & 52 & 2 & 38 & 4 & 12 & 3 & 12 & 3 \\
\hline Pleuroscopia & 4 & 0 & 20 & 3 & 10 & 2 & 2 & 0 & 2 & 0 \\
\hline Pleuroscopia & 12 & 3 & 25 & 3 & 15 & 0 & 3 & 0 & 10 & 4 \\
\hline Pleuroscopia & 4 & 0 & 20 & 6 & 7 & 0 & 3 & 0 & 4 & 0 \\
\hline Pleuroscopia & 4 & 0 & 17 & 0 & 10 & 0 & 3 & 0 & 39 & 0 \\
\hline Pleuroscopia & 4 & 0 & 20 & 0 & 10 & 4 & 2 & 0 & 2 & 1 \\
\hline Pleuroscopia & 6 & 0 & 9 & 2 & 6 & 0 & 1 & 0 & 0 & 0 \\
\hline Traqueostomia & 4 & 0 & 20 & 0 & 10 & 3 & 2 & 0 & 2 & 2 \\
\hline Traqueostomia & 9 & 0 & 80 & 23 & 15 & 0 & 5 & 0 & 6 & 2 \\
\hline Traqueostomia & 8 & 2 & 10 & 1 & 6 & 1 & 4 & 2 & 4 & 0 \\
\hline Traqueostomia & 3 & 0 & 14 & 0 & 9 & 0 & 1 & 0 & 2 & 0 \\
\hline Traqueostomia & 7 & 4 & 26 & 22 & 18 & 10 & 5 & 2 & 8 & 4 \\
\hline Traqueostomia & 2 & 0 & 7 & 0 & 6 & 0 & 1 & 0 & 3 & 1 \\
\hline Traqueostomia & 3 & 0 & 14 & 2 & 7 & 1 & 2 & 0 & 2 & 0 \\
\hline Traqueostomia & 10 & 2 & 14 & 3 & 15 & 2 & 4 & 1 & 5 & 1 \\
\hline Total & 281 & 50 & 999 & 181 & 610 & 92 & 251 & 46 & 1192 & 167 \\
\hline
\end{tabular}


avaliando-se apenas 17 cirurgias de médio porte realizadas em um mês ${ }^{3}$.

Quando discutimos os custos envolvidos no processamento de instrumentais cirúrgicos, há uma série de variáveis extremamente dispendiosas, como uma equipe multidisciplinar composta de profissionais que processam o material, médicos infectologistas e cirurgiões, profissionais que coordenam o controle de infecção, áreas físicas específicas e ambientalmente controladas desde a recepção até o armazenamento do material, atendendo às especificações do fabricante do instrumental desde sua aquisição, para seguir rigorosamente suas instruções para limpeza prévia, transporte, descontaminação, inspeção, teste de funcionalidade, embalagem, desinfecção de alto nível e/ ou esterilização, considerando-se ainda a qualidade da água e os equipamentos de proteção individual utilizados por todos esses profissionais ${ }^{5,12}$.

Em estudo com o objetivo de reduzir a quantidade de instrumentais nos conjuntos de adenoamigdalectomia, que eram frequentemente usados por vários cirurgiões, utilizou-se um método prospectivo de melhoria da qualidade pelo Lean Six Sigma, sendo realizado um mapeamento de fluxo desses instrumentais quanto ao seu uso e processamento. Após a intervenção, foi possível reduzir de 52 para 24 instrumentais de adenoamigdalectomia, com redução do tempo de montagem desses conjuntos de 8,4 para 4,7 minutos ( $\mathrm{p}<0001$ ) e decréscimo de $44 \%$ no custo de montagem dos instrumentais, representando uma estimativa de redução de custos de US $\$ 1.468,99$ por conjunto ${ }^{13}$.

Vale considerar que, nesse mesmo estudo, foram avaliados 700 procedimentos de adenotonsilectomias em um ano e utilizados 850 conjuntos de instrumentais processados no mesmo ano, sendo que os alvos das intervenções para a redução dos custos pelo método Lean foram: tempo perdido entre os passos do processamento dos instrumentais, transporte de componentes desnecessários, instrumentais supérfluos e não utilizados durante o procedimento, atividades desnecessárias dos componentes de processamento e processamento desnecessário dos instrumentais não utilizados ${ }^{13}$.

Assim, a verificação de instrumentais não utilizados em determinados procedimentos constitui uma valiosa ferramenta gerencial que fornece subsídios importantes, visando a redução de custos com o processamento do instrumental cirúrgico, assim como a padronização dos conjuntos de instrumentais para determinados procedimentos, fortemente recomendada desde que haja número e tipo mínimos de instrumentos em cada conjunto5.

Os instrumentais utilizados na categoria "outros" dos tempos cirúrgicos (correspondendo à cirurgia propriamente dita) apresentaram maior taxa de não utilização em relação aos instrumentais dos demais tempos cirúrgicos, pois são também os instrumentais em maior número na composição das caixas avaliadas. Tal fato pode ser atribuído a uma padronização dos conjuntos de instrumentais específicos para determinados procedimentos que não considerou uma composição mínima recomendada pela literatura ${ }^{5,13}$.

Foram verificadas também maiores taxas de não utilização de instrumentais para hemostasia na composição das caixas cirúrgicas, o que pode ser atribuído ao uso de novas tecnologias, como o bisturi elétrico, que apresenta não só a função de corte de tecidos, substituindo o bisturi manual, mas também a função de hemostasia, substituindo os instrumentais de hemostasia por ser, acima de tudo, mais seguro ${ }^{14}$.

Tal fato não ocorreu com os instrumentais de síntese, que estão nessas caixas cirúrgicas em menor quantidade e em proporções necessárias.

As salas cirúrgicas são unidades hospitalares que demandam altos custos e recursos. Em estudo realizado com o objetivo de melhorar o processo cirúrgico multidisciplinar, foi construído um mapa de fluxo de todo o processo cirúrgico, constituído por racionalização do processo pré-operatório; redução de tempo não operatório; eliminação de informações redundantes; e promoção do envolvimento de todos os funcionários. As melhorias dos processos foram implementadas sequencialmente em especialidades cirúrgicas. As principais medidas de desempenho foram coletadas antes e depois da implementação, resultando no aumento da eficiência e na melhoria do desempenho financeiro. Uma das ações realizadas nesse processo foi a descrição minuciosa do procedimento cirúrgico, que permitiu prever e prover criteriosamente os materiais e insumos utilizados em SO, reduzindo consideravelmente o tempo de permanência do paciente e da equipe em $\mathrm{SO}^{15}$.

Os autores observam ainda que o mapeamento de processos constituído por uma equipe multiprofissional de anestesistas, cirurgiões, enfermeiros anestesistas, enfermeiros, pessoal de saúde aliado, administradores de hospitais e analistas de sistemas permitiu o processamento de informações, o suporte de liderança, o engajamento dos funcionários e a implantação de medidas de desempenho eficazes, elementos importantes para melhorar a eficiência de uma sala de cirurgia, garantindo ganhos de desempenho substanciais, sustentáveis e financeiramente positivos ${ }^{15,16}$.

Em estudo que objetivou identificar os tipos, as quantidades e o custo dos materiais de consumo desperdiçados nas cirurgias no intraoperatório em um CC de um hospital universitário de São Paulo, foram avaliados 105 tipos de materiais de consumo em 275 cirurgias observadas em um período de 
quatro meses. Identificou-se que os itens mais desperdiçados foram os fios cirúrgicos e as compressas de gaze, com custo total de $\mathrm{R} \$ 709,84$. O estudo concluiu que o gerenciamento eficiente dos recursos materiais reduz o custo dos processos e diminui os desperdícios ${ }^{17}$.

Um estudo realizado no CC de um hospital público de ensino em Belém (PA), no período de junho a agosto de 2014, identificou que os materiais de insumo mais desperdiçados durante as cirurgias foram os turbantes (15\%), as compressas de gaze (13\%), os medicamentos (12\%) e as luvas (11\%), sendo possível concluir que o desperdício de materiais tem origem estrutural e gerencial e que seu combate requer profunda mudança comportamental dos profissionais, além de rigorosa reestruturação do sistema de distribuição de materiais para o CC. O estudo sugere a implantação de kits cirúrgicos específicos para os procedimentos realizados no $\mathrm{CC}^{18}$.

Este estudo limitou-se à especialidade de "cirurgia do tórax", não apresentando variáveis como os custos gerados com materiais não utilizados e não investigando se a utilização ou não desses materiais estava relacionada às equipes cirúrgicas da especialidade. Contudo, a pesquisa forneceu importantes subsídios que podem ser identificados em estudos futuros e estendidos a outras especialidades que atuam no CC estudado.

\section{CONCLUSÃO}

Este estudo permitiu identificar quantitativamente os instrumentais que não são utilizados em cirurgias torácicas e revelou a necessidade de reformular a composição excedente de instrumentais das caixas cirúrgicas, visando a redução de custos com o processamento de instrumentais que compõem estas caixas e não são utilizados durante os procedimentos.

As instituições poderão reduzir custos com o processamento de instrumentais por meio da revisão dos processos de trabalho, que envolve a participação da equipe multiprofissional atuante no CC no que diz respeito à utilização de instrumentais cirúrgicos.

Com base nos resultados deste estudo, estão sendo implementados a reestruturação da composição de caixas cirúrgicas dessa especialidade e o levantamento dos custos com o processamento de materiais no CME e no CC dessa instituição, visando a organização dos processos de trabalho envolvendo a gestão de instrumentais cirúrgicos.

\section{REFERÊNCIAS}

1. Sociedade Brasileira de Enfermagem de Centro Cirúrgico, Recuperação Anestésica e Central de Materiais e Esterilização. Práticas Recomendadas. 6a ed. São Paulo: SOBECC; 2013.

2. Araruna $A B$, Posso MBS. Centro de material de esterilização: parâmetros espaciais e riscos físicos. Rev SOBECC. 2014 [citado em 2015 out 30];19(3):142-7. Disponível em: http://sobecc.org.br/ arquivos/artigos/2015/pdfs/site_sobecc_v19n3/05_sobecc.pdf

3. Paula JRA, Silva RCR, Vedovato CA, Boaventura AP. Instrumentais nas Caixas Cirúrgicas: Avaliação de Custo. Rev SOBECC. 2015 [citado em 2016 ago 14];20(2):73-80. Disponível em: http://files.bvs.br/ upload/S/1414-4425/2015/v20n2/a5019.pdf

4. Agência Nacional de Vigilância Sanitária. Resolução RDC n. ${ }^{\circ} 30$, de 15 de fevereiro de 2006. Dispõe sobre o registro, rotulagem e re-processamento de produtos médicos, e dá outras providências. Diário Oficial da União. 2006 [citado 2015 out 30]. Disponível em: http://www.aeap.org.br/doc/ resolucao_rdc_30_de_15_de_fevereiro_de_2006.pdf

5. Rothrock JC. Alexander: Cuidados de Enfermagem ao Paciente Cirúrgico. 14 ed. Rio de Janeiro: Elsevier; 2011. 1272p.

6. Freitas LR, Tipple AFV, Pires FV, Melo DS, Spagnoli JLU. (Des)cuidado com produtos para saúde processados no transporte e armazenamento em unidades de internação. Texto Contexto Enferm. 2015 [citado 2016 out. 30];24(1):253-62. Disponível em: http://www.scielo.br/scielo. php?script=sci_arttext\&pid=S0104-07072015000100253

7. Castilho V, Jericó MC. Gerenciamento de custeio: Aplicação de Método de Custeio Baseado em Atividades em Central de Materiais e Esterilização. Rev Esc Enferm USP. 2010 [citado 2015 out 30];44(3): 745-52. Disponível em: http://www.scielo.br/pdf/reeusp/v44n3/28.pdf

8. Schutz V, Siqueira BT. A enfermagem e o custo com os materiais hospitalares: uma revisão bibliográfica. Cogitare Enfermagem. 2011 [citado 2015 out 30];16(1):148-53. Disponível em: http://revistas.ufpr. $\mathrm{br} /$ cogitare/article/viewFile/21126/13952

9. Moresca LGN, Fonseca LF, Tramontini CC. Avaliação dos índices de velocidade e confiabilidade de materiais reprocessados em um centro de material e esterilização. Rev SOBECC. 2011;16(2):34-41.

10. Moriya T, Vicente Y, Tazima MFGS. Instrumental Cirúrgico. Rev Esc Med USP. 2011 [citado 2016 ago. 14];44(1):18-32. Disponível em: http://www.revistas.usp.br/rmrp/article/view/47319/51055

11. Conselho Nacional de Saúde (Brasil). Resolução n. ${ }^{\circ}$ 466, de 12 de dezembro de 2012. Diretrizes e Normas Regulamentadoras de Pesquisas envolvendo Seres Humanos. Diário Oficial da União. 2012 [citado 2015 out 30]. Disponível em: http://bvsms.saude.gov.br/bvs/ saudelegis/cns/2013/res0466_12_12_2012.html 
12. AORN. Guideline for cleaning and care of surgical instruments. In: AORN. Guidelines for Perioperative Practice. 2015 [citado 2017 mar 18]. p.615-50. Disponível em: https://www.aorn.org/websitedata/ cearticle/pdf_file/CEA15517-0001.pdf

13. Wannemuehler TJ, Elghouche AN, Kokoska MS, Deig CR, Matt BH. Impact of Lean on Surgical Instrument Reduction: Less Is More. Laryngoscope. 2015 [citado 2017 mar 18];125(12):2810-5. Disponível em: http://onlinelibrary.wiley.com/doi/10.1002/lary.25407/abstract

14. Olimpio MAC, Sousa VEC, Ponte MAV. 0 uso do bisturi elétrico e cuidados relacionados: revisão integrativa. Rev SOBECC. 2016 [citado 2017 mar 9];21(3):154-61. Disponível em: https://revista.sobecc.org. $\mathrm{br} /$ sobecc/article/view/33

15. Cima RR, Brown MJ, Hebl JR, Moore R, Rogers JC, Kollengode A, et al. Use of Lean and Six Sigma methodology to improve operating room efficiency in a high-volume tertiary-care academic medical center. J Am Coll Surg. 2011 [citado 2017 mar 18];213(1):83-92. Disponivel em: HTTPS://www.ncbi.nlm.nih.gov/pubmed/21420879

16. Ouriques CM, Machado ME. Enfermagem no processo de esterilização de materiais. Texto Contexto Enferm. 2013 [citado 2017 mar 10]; 22(3):695-703. Disponível em: http://www.scielo.br/scielo. php?script=sci_arttext\&pid=S0104-07072013000300016\&lng=pt \&nrm=iso

17. Castro LC, Castilho V. O custo de desperdício de materiais de consumo em um centro cirúrgico. Rev Latino-Am Enferm. 2013 [citado 2017 mar 9];21(6):1228-34. Disponível em: http://www.scielo.br/pdf/rlae/ v21n6/pt_0104-1169-rlae-21-06-0122/.pdf

18. Silva MJN, Ribeiro AL. Gestão em centro cirúrgico: identificação de desperdícios. Rev SOBECC. 2016 [citado 2017 mar. 09];21(2):82-9. Disponível em: https://revista.sobecc.org.br/sobecc/article/ view/120 